\title{
Optimization of Power to Achieve Intended Capacity in Inter-cell Interference of Visible Light Communication Network
}

\author{
Agha Yasir Ali*, Rafay Muhammad Khan, Umm E Laila, Muhammad lbrar-ul-Haque and \\ Amber Israr
}

\author{
Sir Syed University of Engineering and Technology, Karachi, Karachi City, Sindh, Pakistan; aghayasirali@hotmail.com
}

\begin{abstract}
In this article, a novel algorithm is proposed to eliminate the Inter-Cell Interference (ICI) for Visible Light Communication (VLC). The proposed algorithm is based on Hadamard Code (HC) and Pulse Amplitude Modulation (PAM). Four schemes of $\mathrm{HC}_{\mathrm{s}}$ distribution between nine cells are separately designed and tested within a room to mitigate the ICI, whereas PAM is used to improve data rate. The primary objective of this research is to evaluate the amount of power utilized by four proposed schemes to obtain the Channel Capacity Without Interference (CCWI). However, the higher order of PAM (HOP) uses high average power. This increases the ICI which is diminished by using additional power to achieve the CCWI. Therefore, predetermined power is derived for the receivers based on ICI. Moreover, theoretical SER is derived to achieve the trade-off between SER performance and flickering in the room. This is done by choosing positive and negative amplitude from DC bias (PND). The designed four schemes are validated by using computer-based simulation on MATLAB for VLC. The simulated results show that the overall performance of the $4^{\text {th }}$ scheme is power efficient as compared to other three schemes.
\end{abstract}

Keywords: Hadamard Code, Inter-cell Interference, Visible Light Communication

\section{Introduction}

In the recent years, Visible Light Communications (VLC) emerge as the promising technology used to support communication systems in special scenarios such as Hospitals, Conference rooms, and Universities. Many indoor applications for this technology have been suggested which have different challenges ${ }^{1}$. The ICI is one of the significant issues for VLC system. The ICI is caused when the receiver in different neighbour cells attempt to use the same resource at the same time. Various interference avoidance techniques have been studied in the literature under various environmental conditions and network structures. Most common methods are based on the distribution of multi-colour, frequency reuse, time division, cell coordination; however, pilots are used to estimates ICI. Moreover, the power, bandwidth, and blanking resources are utilized to counter the effect of ICI.
$\mathrm{In}^{2}$, the static and dynamic allocation of time, power and frequency resources to receiver is controlled to ensure that the ICI remains within acceptable limits. The proposed scheme in ${ }^{3}$ is comprised of a two-level algorithm - one at the base station level, and another at a central controller to which a group of neighbouring base stations are connected. A polynomial time distributed algorithm is proposed in ${ }^{4}$ to minimize the ICI, the problem of resource allocations has tackled with blanking. However, the excessive use of blanking may deteriorate the performance. The pilot based interference estimation scheme proposed in $^{\frac{5}{}}$ is a crucial step to control power and frequencies resources for cell-edges users. However, the pilot based schemes are not accurate, and it utilizes the extra band resources. The multi-color soft frequency reuse-based VLC system proposed in ${ }^{6}$ adopts the interference coordination, which adjusts the power of the cell-edge components and the cell-centre components. A

*Author for correspondence 
differential polarizer detection system is suggested in $^{7}$ to mitigate the ICI. In this technique, the no of polarizers and photodiodes (PD) is increased with the number of adjacent cells, However, the colors of light in the room are not unique. Moreover, the cell planning has to be strictly well planned. These all proposed schemes are network and cell-based ICI Coordination (ICIC). Rahaim $\stackrel{8}{\text { mitigated }}$ the ICI by using frequency partitioning. The bandwidth is divided into different slots, and neighbouring cells use distinct frequencies to minimize the interference. However, this scheme requires LEDs with a higher bandwidth as the number of cells increase. The authors in ${ }^{9}$, proposed an ICIC and power based mitigation technique, and significantly reduce the computational complexity. However, it uses power constrain which increase the ICI for celledge receivers. The proposed method in $\frac{10}{}$, investigates an angle diversity multi-element based receivers. The ICI is minimized at a certain level but could not be completely eliminated, and it also utilized multiple detectors which is not an economical solution.

This paper investigates a novel scheme to mitigate interference without using multi-color and frequency resources; neither has it required any cell ICIC complexity. It reduces the algorithm complexity for base station and central controller. The proposed technique uses Hadamard codes ${ }^{11}\left(\mathrm{HC}_{\mathrm{s}}\right)$ and the power resources to mitigate ICI and achieve channel capacity without interference (CCWI). The power constrains are utilized in high order PAM (HOP) and it also used to mitigate the ICI effects in cell edges. Therefore, this work reduces the power consumption and mitigates the ICI in cell edges without using power. The power constrains are minimized by utilizing number of $\mathrm{HC}_{\mathrm{s}}$ between cells, as shown in Figure 1. It is becoming increasingly difficult to ignore the utilization of bandwidth by $\mathrm{HC}_{\mathrm{s}}$, which is managed by minimizing the number of $\mathrm{HC}_{\mathrm{s}}$. This is done by reusing them at specific distances in the room as shown in scheme 2 and scheme 3 of Figure 1. The reuse codes between cells can minimize the complexity and bandwidth resources of transceivers. Therefore, a trade-off between ICI, power, bandwidth resources and complexity of the system is achieved. Moreover, the Symbol Error Rate (SER) performance is improved without using additional power by choosing positive and negative amplitude from DC bias (PND). The higher distance between PND improves the SER performance, but it can cause the flickering problem in the room. Therefore, the trade-off between SER performance and flickering is achieved.

This manuscript is organized as follows. Section 2 explains the proposed schemes. The analytical analyses are shown in Section 3. Section 4 represents the simulation results. Section V concludes this paper.

The distribution of HCs in four schemes is designed to minimize the interference, power, bandwidth and $\mathrm{HC}_{\mathrm{s}}$. The transceiver geometry schemes of cell network and the boundaries that separate the cell centre and cell edge region are shown in Figure 1. In these schemes, each cell's receivers are classified as either cell centre or cell-edge depending on their current locations in the room and straight-line distances to the serving cell. The received signals at the receiver can be reported to the cells periodically via the uplink control channels. The channel response $h(t)$ and additive white Gaussian noise (AWGN) $\sigma(t)$ of the VLC system are given in $\mathrm{Eq}(1)$ :

$$
y(t)_{r(x, y, z)}=R h(t) * x(t)+\sigma(t)
$$

where, $y(t)_{r(x, y, z)}$ is the photo-detector current, $R$ represents the responsivity of the photo-detector (in $\mathrm{A} / \mathrm{W})$ and $x(t)$ is the input power. The average transmitted optical power is given in $\mathrm{Eq}(2)$ :

$$
P_{t}=\lim _{T \rightarrow \infty} \frac{1}{2 T} \int_{-T}^{T} x(t) d t
$$

The average received optical power generally can be determined in $\mathrm{Eq}(3)$ :

$$
P_{r}=H_{L O S}(0) P_{t L O S}+\sum H_{r e f}(0) P_{t r e f}
$$

where, the line of sight (LOS) and first reflection channel response is considered that can be express in $\mathrm{Eq}(4)$ and $\mathrm{Eq}(5)$ respectively:

$$
\begin{aligned}
& H(0)_{L O S}=\left\{\begin{array}{c}
\frac{(l+1) A_{P D} \gamma \cos ^{l}(\varphi) g(\psi) T(\psi)_{c o f} \cos (\psi)}{2 \pi d^{2}}, \\
0 \leq \psi \leq \psi_{c} . \\
0, \text { elsewhere }
\end{array}\right\} \\
& H(0)_{r e f}=\left\{\begin{array}{c}
\frac{(l+1) A_{P D} \gamma \cos ^{l}(\varphi) g(\psi) T(\psi)_{\operatorname{cof}} \cos (\psi)}{2 \pi D_{1}^{2} D_{2}^{2}} \\
0 \leq \psi \leq \psi_{c} . \\
0, \text { elsewhere }
\end{array}\right] \cos (\alpha) \cos (\beta),
\end{aligned}
$$


$d$ is the distance between the transmitter and receiver $D_{1}$ is the distance between transmitter

$\mathrm{D}_{2}$ is the distance between reflective point and receiver.

$\gamma$ is the reflectance factor; $d A$ is the reflective area of the small region.

$\alpha$ is the angle of irradiance to the receiver,

$\beta$ is the angle of incidence to the receiver

$l$ is the order of Lambertian emission

$A_{P D}$ is the receiving area

$\varphi$ is the irradiance angle, $\psi$ is the angle of incidence.

$T(\psi)_{\text {cof }}$ is the signal transmission coefficient of an optical filter.

$\psi_{c}$ is the FOV, $p$ is the refractive index.

$g(\psi)$ is the gain of the optical concentrator which is expressed in $\mathrm{Eq}(6) 1$.

\begin{tabular}{|c|c|c|}
\hline \multicolumn{3}{|c|}{ Scheme 1} \\
\hline $\begin{array}{l}\mathrm{Cell}_{7} \\
\mathrm{HC}_{l}\end{array}$ & $\begin{array}{l}\mathrm{Cell}_{\delta} \\
\mathrm{HC}\end{array}$ & $\begin{array}{l}\text { Cello } \\
H_{l}\end{array}$ \\
\hline $\begin{array}{l}\mathrm{Cell}_{4} \\
\mathrm{HC}_{3}\end{array}$ & $\mid \begin{array}{cc}E d_{2} & E d_{3} \\
& \mathrm{Cell}_{5} \\
& \mathrm{HC}_{1} \\
\mathrm{E} d_{1} & \mathrm{E} d_{4}\end{array}$ & $\begin{array}{l}\mathrm{Cell}_{6} \\
\mathrm{HC}_{l}\end{array}$ \\
\hline $\begin{array}{l}\mathrm{Cell}_{3} \\
\mathrm{HC}_{I}\end{array}$ & $\begin{array}{l}\mathrm{Cell}_{2} \\
\mathrm{HC}_{1}\end{array}$ & $\begin{array}{l}\mathrm{Cell}_{3} \\
\mathrm{HC}_{1}\end{array}$ \\
\hline
\end{tabular}

\begin{tabular}{|c|c|c|}
\hline \multicolumn{3}{|c|}{ Scheme 3} \\
\hline $\begin{array}{l}\mathrm{Cell}_{7} \\
\mathrm{HC}_{7}\end{array}$ & $\begin{array}{l}\mathrm{Cell}_{\delta} \\
\mathrm{HC}_{3}\end{array}$ & $\begin{array}{l}\mathrm{Cell}_{9} \\
\mathrm{HC}_{l}\end{array}$ \\
\hline $\begin{array}{l}\mathrm{Cell}_{4} \\
\mathrm{H} \mathrm{C}_{2}\end{array}$ & $\begin{array}{cc}E d_{2} \quad E d_{3} \\
\mathrm{Cell}_{5} \\
\mathrm{HC}_{4} \\
\mathrm{Ed} \quad \mathrm{Ed}_{4}\end{array}$ & $\begin{array}{l}\mathrm{Cell}_{6} \\
\mathrm{HC}_{2}\end{array}$ \\
\hline $\begin{array}{l}\mathrm{Cell}_{3} \\
\mathrm{HC}{ }_{I}\end{array}$ & $\begin{array}{l}\mathrm{Cell}_{2} \\
\mathrm{HC}_{3}\end{array}$ & $\begin{array}{l}\mathrm{Cell}_{3} \\
\mathrm{HC}_{l}\end{array}$ \\
\hline
\end{tabular}

Figure 1. Proposed schemes.

$$
g(\psi)=\left\{\begin{aligned}
\frac{p^{2}}{\sin ^{2} \psi_{c}}, & 0 \leq \psi \leq \psi_{c} \\
0, & 0 \geq \psi_{c}
\end{aligned}\right.
$$

The different cells are transmitting different bit streams using HC. Let's consider the $m$ is the nearest receiver to the $k^{\text {th }}$ cell. Thus, the maximum signal to noise ratio (SNR) is received from the $k^{\text {th }}$ cell. The powers received from others cells which are using the same $\mathrm{HC}$ are considered as

$$
\begin{aligned}
& \text { the signal to noise interference ratio (SNIR) } \sum_{\substack{n=1 \\
n \neq k}}^{N} P_{r(n, m)} \\
& P_{r(m)}=P_{r(k, m)}+\sum_{\substack{n=1 \\
n \neq k}}^{N} P_{r(n, m)}
\end{aligned}
$$

This SNIR power is sent back to the transmitter by uplink channel for the purpose of driving the optimum power. The power resource and HCs are used to mitigate the interference effects and maintain CCWI in all schemes. The reuse of the same HC in neighbour cells causes the high interference. The scheme 4 consumes minimum power because the interference power is eliminated due to the orthogonality of $\mathrm{HC}_{\text {s. }}$ The SNIR is only occurring between cell $_{1}$ and cell $_{9}$. Therefore, negligible additional power is required to mitigate the interference effects. The interference occurs between cell $_{1}$, cell $_{3}$, cell ${ }_{5}$,

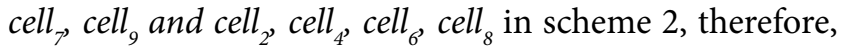
additional power allocation is used between these cells to mitigate the interference effects. Similarly, in scheme 3 cell1, cell3, cell7, and cell9 are sharing $\mathrm{HC}_{1}$, cell $_{2}$, cell $_{8}$, and cell $_{4}$ cell 6 are sharing $\mathrm{HC}_{2}, \mathrm{HC}_{3}$ respectively. The maximum interference occurs in scheme 1 because all neighbour cells are using the same HC. Therefore, it utilizes maximum power. The number of $\mathrm{HC}_{s}$ utilized is translated in additional bandwidth utilization which can be measured as the inverse of shortest time duration peak pulse $\tau_{\min } \underline{12}$ as given in $\mathrm{Eq}(8)$.

$$
W_{x(t)_{n}}=\frac{1}{\tau_{\min }}
$$

Every receiver use $N$ number of matched filters (MF) to receive the signals. However, $N$ depends on total number of $\mathrm{HC}_{\mathrm{s}}$ used in the room. In scheme 1 only one MF is used. Two MFs are used in scheme 2 wherein scheme 3 and scheme 4 are using four and eight numbers of MFs respectively. The principle cell can be recognized by the highest power among number of MFs for the particular receiver. In case of mobile receivers, the $N$ numbers of 
MFs for $N$ numbers of HCs are required to receive signals. However, the receivers with fixed location use only one MF.

\section{Analysis}

The interference signals are dominant in cell edges; therefore, capacity and SER performance are affected in interference areas of the room. The ICI is mitigated by $\mathrm{HC}_{s}$ and CCWI is achieved by the power optimization for each cell in four schemes.

The overall transmission power of each cell is divided into two parts which are cell-edge and cell-center. Let $P_{r(k, E)}$ and $P_{r(k, C)}$. denote the power allocated to cell edge and cell-center then the total power from $k^{\text {th }}$ cell is $P_{r(k, E)}+P_{r(k, C)}=P_{\max }$. Note that $P_{\max }$ is assumed to be maximum possible power faor indoor room environments and it is different for every proposed scheme. The $P_{r(x, y)}$ is required receiving power to obtain CCWI at $(x, y)$ location. The interference power is about negli gible at nearest locations of the cell $\sum_{n=1}^{N} P_{r(n, C)} \cong 0$. The channel capacity, after considering SNIR and bandwidth of $\mathrm{HC}$ is expressed in Eq (9)

$$
C=\frac{q W_{x(t)_{k}}}{2} \log \left(1+\frac{\left(P_{r(k, E)}\right)^{2}}{\left(\dot{o}^{2}+\left(\sum_{\substack{n=1 \\ n \neq k}}^{N} P_{r(n, E)}\right)^{2}\right) q W_{x(t)_{k}}}\right)
$$

where, define the coefficient of bandwidth $W$ which has different value with the different HC is $x(t)_{k}$. Therefore, different schemes use different bandwidth.

The distance and interference are two factors which affect the channel capacity and SER. The interference effects are mitigated by using additional power for receivers. Therefore, the optimum power is derived for interference area to achieve the CCWI. The capacity of interference and non-interference area is compared as follow to achieve required power in $\mathrm{Eq}(10)$.

$$
\begin{aligned}
& \frac{w}{2} \log \left(1+\frac{P_{r(\mathrm{x}, \mathrm{y})}^{2}}{\sigma^{2} W}\right)=\frac{q W_{x(\mathrm{t}) \mathrm{k}}}{2} \log \left(1+\frac{P_{r(k, E)}^{2}}{\left(\sigma^{2}+\sum_{\substack{n=1 \\
n \neq k}}^{N} P_{r(n, E)}^{2}\right) q W_{x\left(\mathrm{t}_{\mathrm{k}}\right.}}\right) \\
& \log \left(1+\frac{P_{r(k, E)}^{2}}{\left(\sigma^{2}+\sum_{\substack{n=1 \\
n \neq k}}^{N} P_{r(n, E)}^{2}\right) q W_{x()_{k}}}\right)=\frac{W}{q W_{x()_{k}}} *\left(1+\frac{P_{r(\mathrm{x}, \mathrm{y})}^{2}}{\sigma^{2} W}\right) \\
& P_{r(k, E)}^{2}=\left[\left(1+\frac{P_{r(x, y)}^{2}}{\sigma^{2} W}\right)-1\right] \frac{W}{q W_{x()_{k}}}\left(\sigma^{2}+\sum_{\substack{n=1 \\
n \neq k}}^{N} P_{r(n, E)}^{2}\right) q W_{x()_{k}} \\
& P_{r(\mathrm{k}, \mathrm{E})}=\sqrt{\left[\left(1+\frac{P_{r(x, y)}^{2}}{\sigma^{2} W}\right)^{\frac{W}{q W_{x(t) k_{k}}}}-1\right]\left(\sigma^{2}+S N I R\right) q W_{x\left(t_{k}\right.}}
\end{aligned}
$$

The power $P_{r(k, E)}$ in $\mathrm{Eq}(10)$ is required power to achieve CCWI. The bandwidth and power management can be control from the transmitter for different locations of the room. The $P_{r(k, E)}$ cannot be increased from certain maximum luminance limit 700-1000 lux for indoor room environment.

The four proposed schemes are used in different arrangements of $\mathrm{HC}_{\mathrm{s}}$ and utilize different bandwidths. Therefore, power requirements of every scheme are also different from each other. The higher bandwidth minimizes the additional power requirement in interference area of the room to meet the requirements of CCWI. A balance can be achieved between power and bandwidth to obtain required luminance in the room.

For example, Figure 1 shows that the interference occurs in cell $_{1}$, cell $_{2}$ and cell 4 at the $E d_{1}$ for cell $_{5}$ in case of scheme 1 . Similarly $E d_{2}, E d_{3}$ and $E d_{5}$ are affected by their neighboring cells. In case of scheme 2 the two $\mathrm{HC}_{s}$ are used, therefore, the interference occurs in cell $_{5}$ is from cell $_{1}$, cell $_{3}$, cell $_{7}$ and cell 9 at $E d_{1}, E d_{4}, E d_{2}$ and $E d_{3}$ respectively. The interferences from cell $_{2}$, cell $_{4}$, cell ${ }_{6}$ and cell $l_{8}$ are mitigated due to orthogonality. The interference power can't be occur if two cells ' $k$ ' and ' ' are using different $\mathrm{HC}\left(H C_{k} H C_{n},(n \neq k)\right)$ as given in Table 1. 
Table 1. ICI for scheme 2

\begin{tabular}{|l|l|l|}
\hline & Cell & Cell \\
\hline ICI & $\sum_{n=3,5,7,9} P_{r(n)}$ & $\sum_{n=4,6,8} P_{r(n)}$ \\
\hline
\end{tabular}

However, in case of scheme 3 every cell uses different HC from neighbour cells. Therefore, no significant interference occurs, however, the far cells can generate interference in case of high SNR or HOP. The ICI for scheme 3 is given in Table. 2 .

Table 2. ICI for scheme 3

\begin{tabular}{|l|l|l|l|l|}
\hline & Cell $_{1}$ & Cell $_{2}$ & Cell $_{4}$ & Cell $_{5}$ \\
\hline ICI & $\sum_{n=3,7,9} P_{r(n)}$ & $P_{r(8)}$ & $P_{r(6)}$ & 0 \\
\hline
\end{tabular}

Similarly, in case of scheme 4 only cell ${ }_{1}$ and cell ${ }_{9}$ are using the same HC. Therefore, no significant interference occurs.

The HOP uses the high average power which creates more interference. A symbol power of $A$-PAM increases with order $A$, where $A$ represents the number of PAM symbols. The symbol power $E_{b} \log _{2}(A)$, of PAM, is substituted in $\mathrm{Eq}(10)$ as follow to achieve required power for $\mathrm{HOP}$ in $\mathrm{Eq}(11)$ :

$P_{r(k, E) H O P}=\sqrt{\left[\left(1+\frac{E_{b} \log _{2}(A)^{2}}{\sigma^{2} W}\right)^{\frac{W}{q W_{x()_{k}}}}-1\right]\left(\sigma^{2}+S N I R_{H}\right) q W_{x(t)_{k}}}$

where, $E_{b}$ is bit energy.

$\mathrm{Eq}$ (11) defines the optimum power used for HOP to achieve CCWI. The higher value of $A$ increases $S N I R_{H}$ which consume more additional power to remove interference effects. In case of HOP, the power requirements for CCWI increase more particularly in case of scheme 1 as compare to scheme 2, 3 and 4 . The difference in power requirement between scheme 2 and 3 increase as $A$ increases because the reuse codes between cells are closer in case of scheme 2 and far in case of scheme 3 . However, in case of scheme 4 no significant difference in power requirements for interference removal at higher values of $A$.

The SER performances cannot be compared in different schemes because every scheme uses different power to obtain CCWI. However, in this paper, the SER performance is improved without using additional power by choosing PND. The massive difference $d_{s}$ between positive and negative improve the SER performance, but it can cause the flickering problem in the room. Therefore, the classic trade-off between SER and flickering is considered as per VLC flickering standards ${ }^{6}$. The SER is derived for high order $A$-PAM in Eq(12)

$$
\operatorname{SER}_{(A-P A M)}=\frac{A-1}{A} \operatorname{erfc}\left(\frac{d_{s}}{2} \sqrt{\frac{E_{b} \log _{2}(A)}{\left(\sigma+\sum_{\substack{n=1 \\ n \neq k}}^{N} P_{r(n)}\right) P_{a v g}}}\right)
$$

It is analyzed in $\operatorname{Eq}(12)$ that, the value of $d_{s}$ can be optimized to achieve the minimum acceptable flickering in the room. Moreover, $d_{s}$ is proportional to SER performance and it also producing flickering in the room.

\section{Simulation Result and Discussion}

The setup of the simulation program for indoor VLC system is shown in Figure 1, where nine cells located on the ceiling and simulation program for SER, capacity and optimum power has taken of $5 m \times 5 m \times 3 m$ room, and the simulations are plotted from $(1,1)$ location to $(6,6)$ location. The four schemes are compared to achieve the equal distribution of channel capacity as shown in Figure 2. The channel capacity and SER performance deteriorate in cell edges due to ICI and distance. Therefore, addition power has plotted to mitigate the ICI effects for cell-edge receivers.

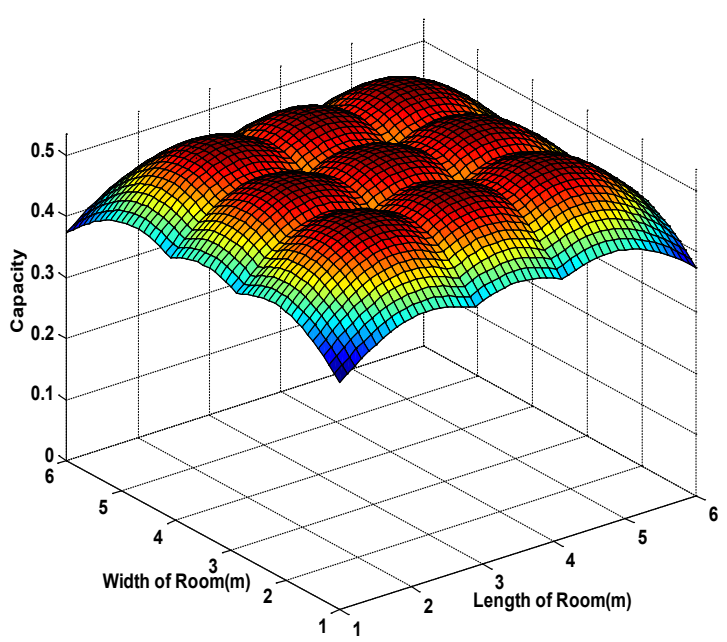

Figure 2. Channel capacity CCWI. 
Figures 3 and 4 show the two and three dimensional comparison of the optimum power of four schemes by obtaining the capacity as shown in Figure 2. Note that the high power is required at the centre and cell edges of the room. The scheme 1 utilizes the highest power then scheme 2, 3 and 4 as shown in Figure 3. The scheme 2 and 3 have the minor difference because both schemes use different $\mathrm{HC}_{\mathrm{s}}$ in neighbour cells. However, the scheme 3 reuse the $\mathrm{HC}_{\mathrm{s}}$ at far distance as compared to scheme 2 . The scheme 4 uses very less power to obtain capacity in Figure 2, because almost every cell use the different HC so the receivers can easily distinguish the principle signal and interference signal. Therefore, the negligible power is used to mitigate the interference.

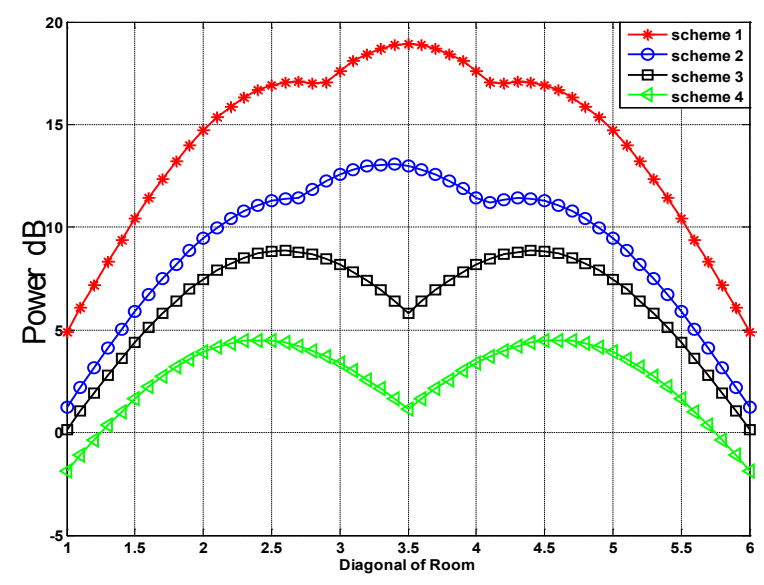

Figure 3. Comparison of power to obtain CCWI.
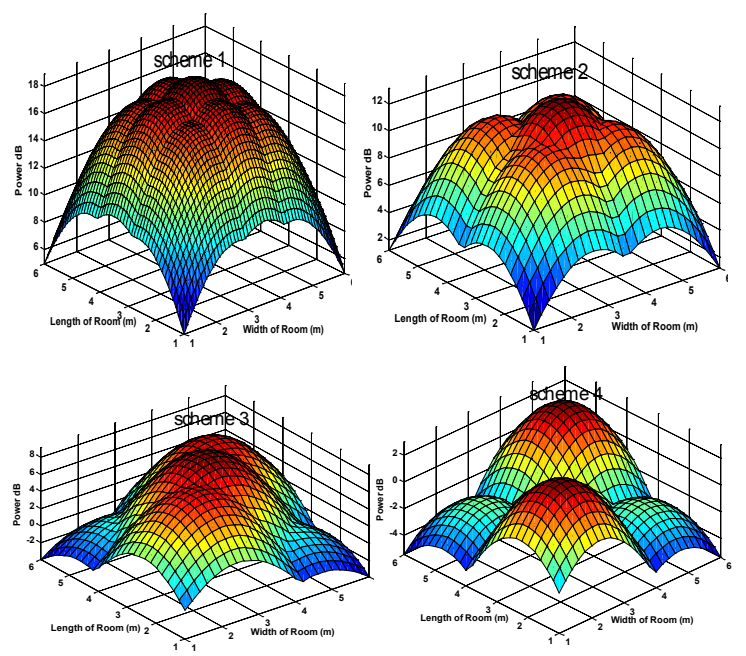

Figure 4. Three dimensional representation of power requirements for scheme $1-4$.

To produce the same capacity for all cells, the Figure 4 shows the similar additional power for all cells edges in scheme 1, because same HC is used by all cells. However, in scheme 2 the cell $_{2}$, cell ${ }_{4}$, cell ${ }_{6}$ and cell $_{8}$ are using high bandwidth HC then cell $_{1}$, cell ${ }_{3}$, cell ${ }_{5}$, cell ${ }_{7}$ and cell, therefore high bandwidth $\mathrm{HC}_{\mathrm{s}}$ has utilized less power than low bandwidth $\mathrm{HC}_{s}$. Similarly, in scheme 3 the cell $_{1}$, cell $_{5}$ and cell $_{9}$ are using low bandwidth $\mathrm{HC}$ and use higher power than other cells. Note that the scheme 4 in Figure 4, the cell $l_{1}$ and cell $_{9}$ are sharing low bandwidth $\mathrm{HC}$ and use high power to meet the capacity requirement in Figure 2. We conclude that high bandwidth $\mathrm{HC}_{s}$ and less reuse of $\mathrm{HC}_{s}$ save the power significantly. In this paper, the optimum power has compared by abstaining the required channel capacity CCWI for four schemes.

Figure 5 shows the overall comparison of the average power of 50X50 receiver's locations in cells centers and cell-edges of the room. This comparison shows the difference of all schemes in all possible areas of the receivers.

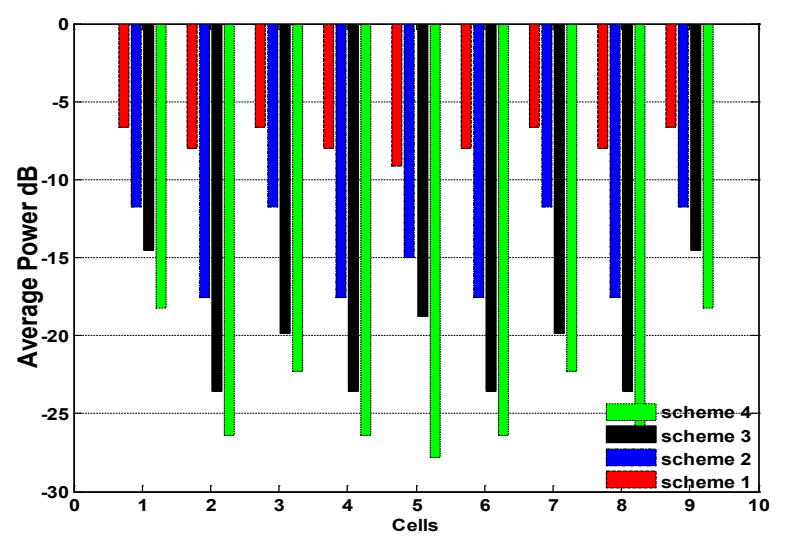

Figure 5. Average received power for 50X50 receiver's locations.

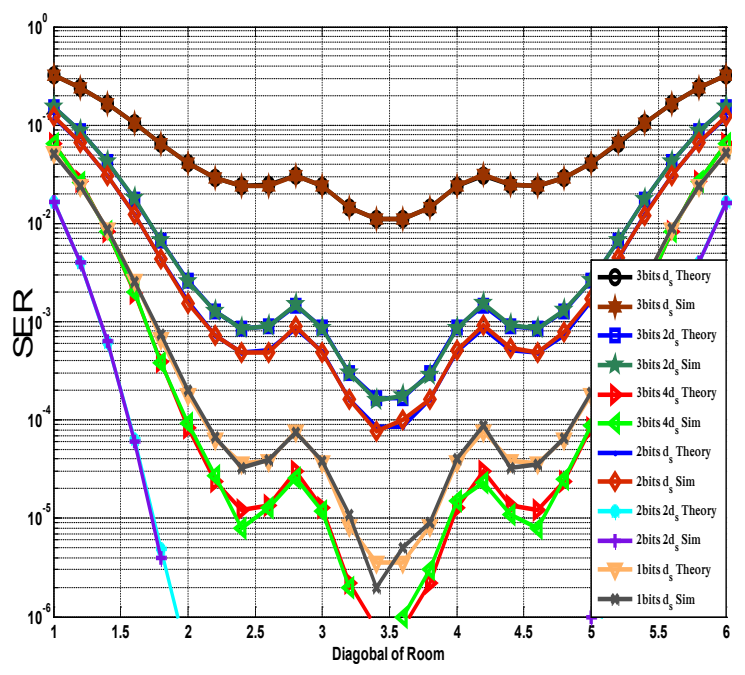

Figure 6. SER comparison for different values of $d_{s}$. 
Finally, the theoretical and simulation SER are plotted in Figure 6. The different order of PAM is simulated under the scheme 1 environment at $S N R=60 \mathrm{~dB}$, and results show that the theoretical and simulation SER performances are identical. The amplitude levels are separated with $d \quad 10 d B$, and the higher value of $d_{s}$ produces better SER performances.

\section{Conclusion}

In this paper, the power efficiency and SER performances are investigated in ICI environments of VLC system. The four schemes of $\mathrm{HC}_{\mathrm{s}}$ distribution between nine cells are compared in term of power to achieve CCWI. The results show that the frequent reuse of $\mathrm{HC}_{\mathrm{s}}$ in cells increase the power requirements to mitigate the ICI effects. For Example, the scheme 1 uses more power because only one $\mathrm{HC}$ is reused in all cells. However, the scheme 2 utilizes low power then scheme 1 because two $\mathrm{HC}_{\mathrm{s}}$ are reused in nine cells. Similarly, scheme 3 utilizes four $\mathrm{HC}_{\mathrm{s}}$ which are reused in nine cells and provide better power efficiency than scheme 1 and 2. The scheme 4 uses maximum eight $\mathrm{HC}_{s}$ and utilize minimum power than all schemes.

The SER performance is also improved without using additional power by choosing PNA from DC bias. The trade-off between SER performance and flickering in the room is achieved.

\section{Acknowledgement}

The author(s) declare(s) that there is no conflict of interest regarding the publication of this paper. This paper is financially supported by National High Technology Rese arch and Development Program of China (863 Program: 2013AA014000).

\section{References}

1. Komine T, Nakagawa M. Fundamental analysis for visible-light communication system using LED lights. IEEE Transactions on Consumer Electronics. 2004; 50(1):100-7. https://doi.org/10.1109/TCE.2004.1277847

2. Hamza AS, Khalifa SS, Hamza HS, Elsayed K. A survey on inter-cell interference coordination techniques in OFDMAbased cellular networks. Communications Surveys and
Tutorials. 2013; 15(4):1642-70. https://doi.org/10.1109/ SURV.2013.013013.00028

3. Rahman M, Yanikomeroglu H. Enhancing cell-edge performance: A downlink dynamic interference avoidance scheme with inter-cell coordination. IEEE Transactions on Wireless Communications. 2010; 9(4):1414-25. https://doi. org/10.1109/TWC.2010.04.090256

4. Sediq AB, Schoenen R, Yanikomeroglu H, Senarath G. Optimized distributed Inter-cell Interference Coordination (ICIC) scheme using projected subgradient and network flow optimization. IEEE Transactions on Communications. 2015; 63(1):107-24. https://doi.org/10.1109/TCOMM. 2014. 2367020

5. Mhatre VP, Rosenberg CP. Impact of network load on forward link inter-cell interference in cellular data networks. IEEE Transactions on Wireless Communications. 2006; 5(12):3651-61. https://doi.org/10.1109/TWC.2006.256988

6. Gomez J, Morcos M. Flicker measurement and light effect. IEEE Power Engineering Review. 2002; 22(11):11-15. https://doi.org/10.1109/MPER.2002.1045556

7. Ryoo H-N, Kwon D-H, Yang S-H, Han S-K. Differential optical detection in VLC for inter-cell interference reduced flexible cell planning. IEEE Photonics Technology Letters. 2016; 28(23):2728-31. https://doi.org/10.1109/ LPT.2016.2615644

8. Rahaim M, Little T. SINR analysis and cell zooming with constant illumination for indoor VLC networks. 2013 2nd International Workshop on Optical Wireless Communications (IWOW); 2013. https://doi.org/10.1109/ IWOW.2013.6777769

9. Adeel A, Larijani H, Ahmadinia A. Resource management and inter-cell-interference coordination in LTE uplink system using random neural network and optimization. IEEE Access. 2015; 3:1963-79. https://doi.org/10.1109/ ACCESS.2015.2489865

10. Zhong, W-D, Chen C, Yang H, Du P. Performance analysis of angle diversity multi-element receiver in indoor multi-cell visible light communication systems. 2017 19th International Conference on Transparent Optical Networks (ICTON); 2017. IEEE. https://doi.org/10.1109/ ICTON.2017.8024792

11. Tarokh V, Jafarkhani H, Calderbank AR. Space-time block codes from orthogonal designs. IEEE Transactions on Information Theory. 1999; 45(5):1456-67. https://doi. org/10.1109/18.771146

12. Ali AY, Zhang Z, Abdelgader AMS, Zong B. Data rate optimization in inter-cell interference environment of visible light communication. Photonic Network Communications; 2015. p. 1-9. https://doi.org/10.1007/s11107-015-0585-0 